\title{
Osteoporosis, bone turnover and hypogonadism in elderly men with treated leprosy
}

\author{
ASAKO ISHIKAWA*, $\dagger$, SATOSHI ISHIKAWA** \& \\ MASAHISA HIRAKAWA $\dagger$ \\ *Department of Anesthesiology, National Leprosarium Nagashima \\ Aisei-en, Japan \\ **Department of Rehabilitation Medicine, Higashi Osaka Hospital, \\ Japan \\ $\dagger$ Department of Anesthesiology and Resuscitology, Okayama \\ University Medical School, Japan
}

Accepted for publication 5 July 2001

\begin{abstract}
Summary In male hypogonadism associated with bone loss, it is important to determine whether bone loss continues with ageing and an increased risk of fracture. We studied bone metabolism in 86 male leprosy patients, who were classified according to the presence or absence of osteoporosis. Osteoporosis was present when men had lumbar compression fractures or a mean BMD-2SD that of normal Japanese men in each age decade. Four men had fractures. Serum concentrations of 1,25-dihydroxyvitamin D and high-sensitivity parathyroid hormone were almost normal in both groups, whereas free testosterone and oestradiol were significantly lower in the osteoporosis group than in the non-osteoporosis group (free testosterone: $P<0.01$, oestradiol: $P<0.05$ ). The urinary concentrations of pyridinoline and deoxypyridinoline, as a marker of bone absorption, were significantly higher in the osteoporosis group than in the non-osteoporosis group (pyridinoline: $P<0.01$, deoxypyridinoline: $P<0.01$ ). The serum concentration of osteocalcin, a marker of bone formation, was significantly higher in the osteoporosis group than in the nonosteoporosis group $(P<0 \cdot 01)$. Elevated concentration means that bone repair is increased possibly because of compensation mechanisms for increased bone loss. In the osteoporosis group, hypogonadism occurred, and high bone turnover continued even in older men. We recommend clinical studies of treatment such as replacement therapy to prevent bone loss and increasing risk of fractures in older men with leprosy.
\end{abstract}

\section{Introduction}

In male hypogonadism, sex hormones (testosterone and oestrogen) are the main factors regulating bone mineral density (BMD). ${ }^{1-5}$ We see male leprosy outpatients with or without

Correspondence to: Asako Ishikawa, Department of Anesthesiology and Resuscitology, Okayama University Medical School, 2-5-1, Shikata-cho, Okayama city, 700-8558, Japan (email: satoshi-2001@mbm.sphere.ne.jp) 
complaints such as back pain and discover osteoporosis in some of them on X-ray. We previously reported that hypogonadism contributes to bone loss in male leprosy patients. ${ }^{6-9}$ We measured the cortical thickness of the 2nd metacarpal bone index in 499 leprosy patients whose ages ranged from 40 to 70 years and more. ${ }^{6}$ The metacarpal bone index of 238 male patients with lepromatous leprosy was lower than that of 139 female patients with lepromatous leprosy. We also measured bone mineral densities in 31 male leprosy patients and 31 age-matched healthy controls, and we found that serum concentrations of free testosterone and oestradiol concentrations were significantly lower and luteinizing hormone concentrations significantly higher in leprosy patients than in controls. ${ }^{7}$ Bone mineral density of the forearm significantly correlated with free testosterone concentrations $(r=0.689$, $P<0.0001)$. We then conducted a mass osteoporosis examination and measured the bone mineral density of 353 leprosy patients (197 men and 156 women) and serum concentrations of free testosterone in 81 of the men. ${ }^{8}$ More than $30 \%$ of men at all ages had osteoporosis. Serum concentrations of free testosterone in men ranged from almost 0 to normal at all ages, and bone mineral density was significantly correlated with serum concentrations of free testosterone. In another study, we used histopathological and histomorphometric methods to compare the degree of testicular damage with that of trabecular bone loss in autopsy cases. ${ }^{9}$ Trabecular bone volume was maintained in patients with nodular Leydig cell hyperplasia in the testes, which secrete androgen hormone, whereas testes without Leydig cell hyperplasia showed a loss of trabecular bone volume. These findings indicated that secondary gonadal dysfunction caused by testicular atrophy may be a factor in osteoporosis in male leprosy patients and that Leydig cell hyperplasia apparently preserves bone volume. From the combined results of these previous studies, we concluded that the orchitis caused by Mycobacterium leprae in their youth contributed to the development of osteoporosis in ageing male leprosy patients.

Today, in Japan patients with leprosy are often in their 70s. In male hypogonadism associated with bone loss, the most interesting question is whether high turnover continues in older men, as it does in postmenopausal women, thereby increasing the risk of fracture.

To study bone turnover, we measured urinary concentrations of pyridinoline (Pyr) and deoxypyridinoline (Dpyr), and serum concentrations of osteocalcin (OC). Urinary Pyr, a cross-linking intercollagen molecule, and Dpyr, an analogue of Pyr, are known as markers of bone resorption. ${ }^{10-14}$ Serum osteocalcin, which is a bone-specific protein secreted by osteoblasts, ${ }^{15}$ serves as an index of bone formation. ${ }^{13,16}$ To study bone metabolism, we compared the serum 1,25-dihydroxyvitamin $\mathrm{D}\left[1,25(\mathrm{OH})_{2} \mathrm{D}\right]$, parathyroid hormone $(\mathrm{PTH})$, and gonadal steroid (free testosterone and oestrogen) concentrations between those with and those without osteoporosis among a group of male leprosy patients.

\section{Materials and methods}

SUBJECTS

Eighty-six male leprosy patients, aged 45-83 years (average: 65.5), agreed to have blood, urine, and bone mineral density tests conducted. They had all lived for several decades at the national leprosarium on a small island in Japan, had eaten almost the same diet, which was calculated to provide a certain amount of calories and nutritional balance, and had normal activities of daily living. None of the patients was receiving androgen replacement therapy or 
steroid therapy before this study. Only five men had been previously treated for osteoporosis (vitamin D replacement by oral or intramuscular calcitonin). Informed consent was obtained from each patient, and we explained the results and treatment plan to all patients. Approval for this study was obtained from our institutional ethics committee.

BMD AND BODY CONSTITUENT DETERMINATIONS

BMD data, in grams per square centimeter, were determined by dual energy X-ray absorptiometry (DXA) with a Hologic QDR-4500 densitometer (Biologic Inc., Waltham, MA, USA). The standard sites checked were the lumbar vertebrae (L2-L4). Kin et al. ${ }^{17}$ measured BMD of 248 healthy Japanese male volunteers grouped according to decade of age. The result was $1.027 \pm 0.084 \mathrm{~g} / \mathrm{cm}^{2}$ in those aged $20-29,1.195 \pm 0.128 \mathrm{~g} / \mathrm{cm}^{2}$ in those aged $30-39,1 \cdot 132 \pm 0 \cdot 101 \mathrm{~g} / \mathrm{cm}^{2}$ in those aged $40-49,1 \cdot 101 \pm 0 \cdot 119 \mathrm{~g} / \mathrm{cm}^{2}$ in those aged $50-59$, $1.091 \pm 0.157 \mathrm{~g} / \mathrm{cm}^{2}$ in those aged $60-69$, and $0.970 \pm 0.162 \mathrm{~g} / \mathrm{cm}^{2}$ in those aged $70-79$ years of age or older. Using their data, we defined below mean BMD-2SD in each age decade or the presence of lumbar compression fractures as indicating osteoporosis and defined above mean BMD-2SD in each decade as indicating non-osteoporosis. Osteoporosis was defined as a BMD-2SD or more below the normal mean BMD (relative to age) or the presence of lumbar compression fractures. The cut-off values of osteoporosis measured at the lumbar vertebrae were $0.930 \mathrm{~g} / \mathrm{cm}^{2}$ in the $40 \mathrm{~s}, 0.863 \mathrm{~g} / \mathrm{cm}^{2}$ in the $50 \mathrm{~s}, 0.777 \mathrm{~g} / \mathrm{cm}^{2}$ in the $60 \mathrm{~s}$, and $0.646 \mathrm{~g} / \mathrm{cm}^{2}$ in the 70 s or older.

\section{HORMONES AND OTHER PROFILES}

Serum concentrations of free testosterone, oestradiol, $1,25(\mathrm{OH})_{2} \mathrm{D}$, and high-sensitivity PTH $(\mathrm{HS}-\mathrm{PTH})^{18}$ were measured by radioimmunoassay (RIA). Urinary Pyr and Dpyr concentrations were measured by high-performance liquid chromatography (HPLC) ${ }^{19}$ in selected patients (Pyr and Dpyr: 25 patients with osteoporosis and 33 without osteoporosis), and serum OC was measured by immunoradiometric assay (IRMA) in 55 patients ( 25 with osteoporosis and 30 without osteoporosis). The values of urinary Pyr and Dpyr in urine samples are expressed per mol of urinary creatinine. All samples were measured with commercial kits (SRL Inc., Tokyo, Japan). Non-fasting blood was drawn between 0900 and $1100 \mathrm{~h}$, and the plasma was removed and stored at $-70^{\circ} \mathrm{C}$ until assay. Intra-assay coefficients of variation determined at our laboratory are $2.22 \%$ Pyr, 3.50\% Dpyr, 3.84\% OC, $2.69 \% 1,25[\mathrm{OH}]_{2} \mathrm{D}$, $6.95 \%$ HS-PTH, $4.23 \% \mathrm{FT}$ and $4.73 \% \mathrm{E}_{2}$. Inter-assay coefficients of variation specified by the manufacturer $2 \cdot 35-3.83 \%$ Pyr, $4 \cdot 23-5.96 \%$ Dpyr, $5 \cdot 05-8.97 \%$ osteocalcin, $3 \cdot 29-5 \cdot 31 \%$ $1,25[\mathrm{OH}]_{2} \mathrm{D}, \quad 10 \cdot 2-12.6 \%$ HS-PTH, $6.63-18.90 \%$ free testosterone and $2 \cdot 32-6 \cdot 10 \%$ oestradiol.

\section{STATISTICAL ANALYSIS}

Statistical analyses were performed with the Stat View package, version 5.0 (SAS Insitute, Cary, NC, USA) on an Apple Macintosh computer. All values are expressed as means \pm SD unless otherwise indicated. Differences between groups were analysed by Mann-Whitney $U$ test (two groups) and Kruskal-Wallis test (three groups). Relations between pairs of variables were analysed by Spearman rank correlation. $P$ values $<0.05$ were considered statistically significant. 


\section{Lumbar (L2-L4) BMD (g/cm $\left.{ }^{2}\right)$}

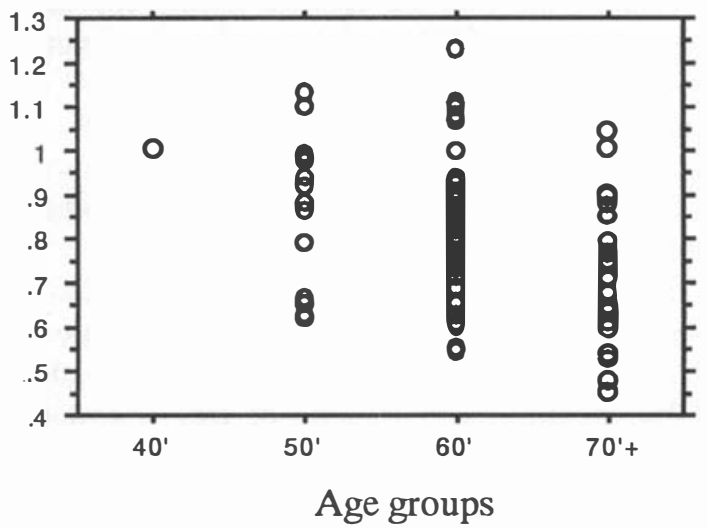

Figure 1. Bone mineral densities in each decade.

\section{Results}

Four men had lumbar compression fractures. Bone mineral density of the osteoporosis group $(n=37)$ was $0.663 \pm 0.094 \mathrm{~g} / \mathrm{cm}^{2}$ and that of the non-osteoporosis group was $0.919 \pm 0.129 /$ $\mathrm{g} / \mathrm{cm}^{2}(n=49)$.

Mean BMD was not significantly different in any age decade (Figure 1 ). The means \pm SD for all measured values in both groups are listed in Table 1. Age matching was done in the two

Table 1. Biochemical data for the osteoporosis and non-osteoporosis groups. BMI = body mass index; Pyr = pyridinoline; Dpyr = deoxypyridinoline; 1,25 $(\mathrm{OH})_{2} \mathrm{D}=1,25$-dihydroxyvitamin $\mathrm{D} ; \mathrm{HS}$ $\mathrm{PTH}=$ high sensitivity of parathyroid hormone; $\mathrm{FT}=$ free testosterone; $\mathrm{E}_{2}=$ oestradiol

\begin{tabular}{lcc}
\hline & $\begin{array}{c}\text { Osteoporosis } \\
(n=37)\end{array}$ & $\begin{array}{c}\text { Non-osteoporosis } \\
(n=49)\end{array}$ \\
\hline Mean age $($ years $)$ & $65 \cdot 8 \pm 5 \cdot 7$ & $65 \cdot 3 \pm 7 \cdot 7$ \\
BMI $\left(\mathrm{kg} / \mathrm{m}^{2}\right)$ & $25 \cdot 1 \pm 3 \cdot 2$ & $24 \cdot 5 \pm 3 \cdot 9$ \\
Pyr $(\mu \mathrm{mol} / \mathrm{mol}$ creatinine $)$ & $29 \cdot 1 \pm 8 \cdot 7$ & $22 \cdot 4 \pm 6 \cdot 8^{* *}$ \\
& $(n=25)$ & $(n=33)$ \\
Dpyr $(\mu \mathrm{mol} / \mathrm{mol}$ creatinine $)$ & $5 \cdot 5 \pm 1 \cdot 7$ & $4 \cdot 2 \pm 1 \cdot 6^{* *}$ \\
& $(n=25)$ & $(n=33)$ \\
Osteocalcin $(\mathrm{ng} / \mathrm{ml})$ & $7 \cdot 96 \pm 3 \cdot 60$ & $5 \cdot 48 \pm 2 \cdot 22^{* *}$ \\
& $(n=25)$ & $(n=30)$ \\
$1,25(\mathrm{OH})_{2} \mathrm{D}(\mathrm{pg} / \mathrm{ml})$ & $38 \cdot 3 \pm 14 \cdot 6$ & $40 \cdot 0 \pm 13 \cdot 0$ \\
& $(n=32)$ & $(n=45)$ \\
HS-PTH $(\mathrm{pg} / \mathrm{ml})$ & $335 \cdot 3 \pm 136 \cdot 4$ & $333 \cdot 3 \pm 91 \cdot 1$ \\
& $(n=32)$ & $(n=39)$ \\
FT $(\mathrm{pg} / \mathrm{ml})$ & $3 \cdot 92 \pm 4 \cdot 01$ & $6 \cdot 99 \pm 4 \cdot 83^{* *}$ \\
& $(n=37)$ & $(n=47)$ \\
$\mathrm{E}_{2}(\mathrm{pg} / \mathrm{ml})$ & $13 \cdot 83 \pm 6 \cdot 14$ & $17 \cdot 40 \pm 9 \cdot 20^{*}$ \\
& $(n=33)$ & $(n=45)$ \\
& & \\
\hline
\end{tabular}

Relative to osteoporosis: $* P<0.05,{ }^{* *} P<0.01$. 
groups. The urinary concentrations of Pyr and Dpyr were significantly higher in the osteoporosis group than in the non-osteoporosis group (pyridinoline: $P<0 \cdot 01$, deoxypyridinoline: $P<0.01$ ) (Figure 2a,b). To minimize the urine sampling error, all data were corrected for urinary creatinine. The serum concentration of $\mathrm{OC}$ was significantly higher in the osteoporosis group than in the non-osteoporosis group $(P<0 \cdot 01)$. (Figure $2 \mathrm{c})$. Since serum concentrations of $\mathrm{OC}$ are cleared by the kidney and serum concentrations can be influenced by renal function, we measured creatinine and confirmed that indices of renal function did not affect serum concentrations of OC.

Serum concentrations of $1,25(\mathrm{OH})_{2} \mathrm{D}$ and HS-PTH were also not significantly different between the groups and were almost the same as the laboratory preference ranges. Serum concentrations of $1,25(\mathrm{OH})_{2} \mathrm{D}$ were negatively correlated with $\mathrm{HS}-\mathrm{PTH}$ concentrations $(r=-0 \cdot 247, P<0 \cdot 05)$. Serum concentrations of free testosterone and oestradiol were signficantly higher in the non-osteoporosis group than in those with osteoporosis (FT: $P<0.01$, oestradiol: $P<0 \cdot 05)$.

\section{Discussion}

Determining how osteoporosis develops in men with leprosy may provide useful information about osteoporosis in general. In our present study, the mean BMDs were below normal in each age group and showed no significant difference between patients in their 50s, 60s and 70 s or older. BMD varied widely within each decade. This suggests that some factor other than ageing regulates bone volume in men with leprosy. We concluded previously that hypogonadism was an important factor.

Patients with leprosy are often in their 70s in Japan, and our interest is in whether diminishing bone volume continues rapidly with ageing in patients with osteoporosis and hypogonadism. If high bone turnover continues, risk of bone fracture must increase, and femur fractures contribute to a high mortality in older men.

Urinary Pyr and Dpyr are markers of bone resorption, and serum OC indicates osteoblastic activity. During high bone turnover such as that observed in postmenopausal women, these markers elevate. ${ }^{13,14}$ In our current study, urinary concentrations of Pyr and Dpyr showed significant differences between the two groups $(P<0 \cdot 01)$. The serum concentration of OC, a marker of bone formation, was significantly higher in the osteoporosis group than in the non-osteoporosis group $(P<0 \cdot 01)$. Elevated OC indicates that bone repair is increased possibly due to compensation mechanisms for increased bone loss. Although the amounts of Pyr, Dpyr and OC were not measured in all subjects, we believe our data indicate that high turnover and bone loss had been ongoing in the osteoporosis group. This situation may be similar to that of postmenopausal women. If this high turnover cannot be corrected, the risk of fracture associated with ageing is greater for osteoporotic men than for nonosteoporotic men.

What can correct this high turnover? In Japan, vitamin D and calcium replacement therapies have been used in older women and men. Because intestinal calcium absorption decreases with ageing due to vitamin D deficiency in women ${ }^{20,21}$ and men, ${ }^{20}$ hypovitaminosis D predisposes individuals to hip fractures. ${ }^{22,23}$ In calcium homeostasis in women, Riggs et al. ${ }^{24}$ reported that type I (postmenopausal) osteoporosis was associated with decreased production of $1,25(\mathrm{OH})_{2} \mathrm{D}$ secondary to a decrease in $\mathrm{PTH}$, which leads to decreased calcium absorption. In type II (senile) osteoporosis, decreased osteoblast function and impaired production of 
(a)

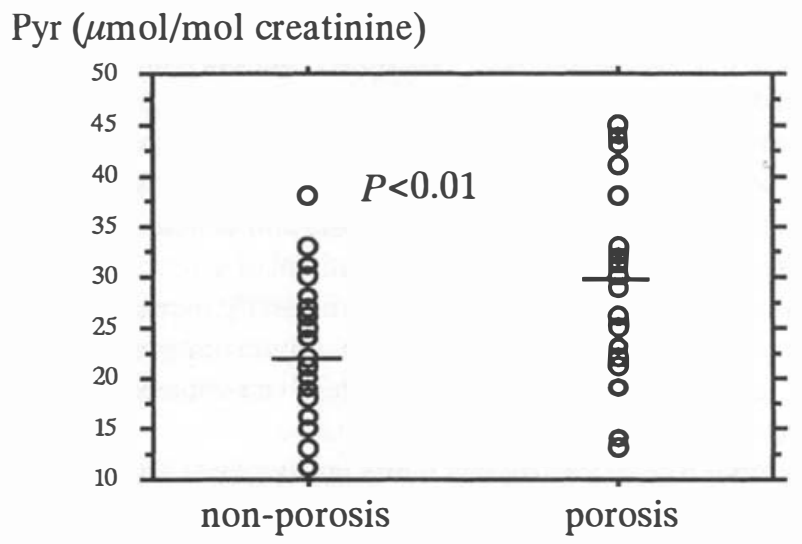

(b)

Dpyr ( $\mu \mathrm{mol} / \mathrm{mol}$ creatinine)

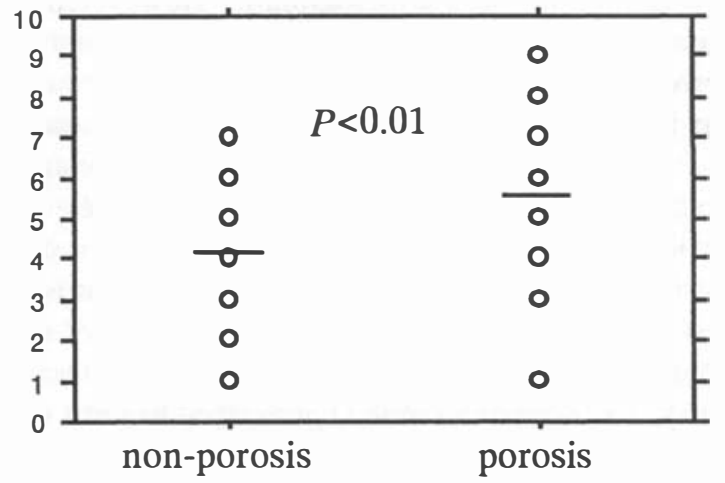

(c)

$\mathrm{OC}(\mathrm{ng} / \mathrm{ml})$

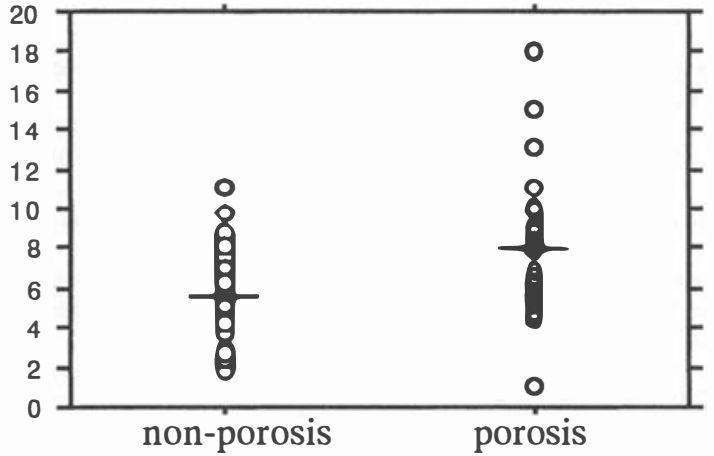

Figure 2. (a) Urinary pyridinoline concentrations in osteoporosis and non-osteoporosis groups. (b) Urinary deoxypyridinoline concentrations in osteoporosis and non-osteoporosis groups. (c) Osteocalcin concentrations in osteoporosis and non-osteoporosis groups. 
$1,25(\mathrm{OH})_{2} \mathrm{D}$ are primary, also leading to decreased calcium absorption and secondary hyperparathyroidism. In men, lower calcium absorption is reportedly associated with reduced plasma $1,25(\mathrm{OH})_{2} \mathrm{D}$ concentrations. ${ }^{2,25}$ However, our present results showed that $1,25(\mathrm{OH})_{2} \mathrm{D}$ and HS-PTH had almost normal concentrations, with no significant differences between the osteoporosis and non-osteoporosis groups. Therefore, the administered dose of vitamin D was sufficient, and further replacement of vitamin D may not be effective. Jackson et al ${ }^{26}$ examined men with idiopathic or hypogonadal osteoporosis and concluded that when hypogonadal men with osteoporosis maintain abundant concentrations of vitamin $\mathrm{D}$, their $1,25(\mathrm{OH})_{2} \mathrm{D}$ synthesis remains normal, and bone remodeling is only modestly increased, which can be corrected by hormone therapy as in postmenopausal women. Given our present results, we agree with their idea. Hormone replacement therapy may be effective to correct bone turnover, even in elderly men.

The physiologial role of oestrogens in the human male has been reported. Two categories of oestrogen disorder have been noted: congenital absence or functional absence of oestrogens. Reports document an aromatase deficiency (aromatase cytochrome P450) ${ }^{27-29}$ and an oestrogen-receptor defect. ${ }^{30}$ In patients with aromatase deficiency, testosterone was normal $^{28}$ or high $^{27,29}$ and oestradiol was low. ${ }^{27-29}$ In patients with oestrogen-receptor defect, testosterone was normal and oestradiol was high. ${ }^{30}$ However, in our present data, concentrations of oestradiol and free testosterone were much lower than normal, and the low concentrations of oestradiol were correlated with those of free testosterone $(r=0.563, P<0.0001)$. The hypogonadism in male leprosy patients is acquired (invasion of testes by Mycobacterium leprae) rather than congenital, and low concentrations of oestrogen may possibly be due to the decreased free testosterone rather than to impaired aromatization due to substrate deficiency for P450 aromatase. Which is dominant, testosterone or oestrogen, remains unclear.

In conclusion, in hypogonadal men with long-term leprosy, high bone turnover continues with ageing. We recommend clinical studies of treatment such as replacement therapy to determine appropriate measures to prevent bone loss and increasing risk of fractures in older men with leprosy. The dose of vitamin D prescribed to some of our patients seemed sufficient to prevent severe bone loss. We believe serum concentrations of FT regulate BMD. We postulate that hormone replacement therapy may be effective even in older men.

\section{References}

1 Baillie SP, Davison CE, Johnson FJ, Francis RM. Pathogenesis of vertebral crush fracture in men. Age Ageing, 1992; 21: 139-141.

2 Francic RM, Peacock, M, Marshall DH et al. Spinal osteoporosis in men. Bone Miner, 1989; 5: 347-357.

3 Jackson JA, Kleerekoper M. Osteoporosis in men: diagnosis, pathophysiology, and prevention. Medicine, 1990; 69: $137-152$

4 Seeman E, Melton LJ III, O’Fallon WM, Riggs BL. Risk factors for spinal osteoporosis in men. Am J Med, 1983; 75: $977-983$.

5 Seeman E. Osteoporosis in men. Bailliere's Clin Rheumatol, 1997; 11: 613-629.

6 Ishida Y, Ishikawa S. Hypogonadal osteoporosis in elderly male patients with lepromatous leprosy. Jpn J Leprosy, 1997; 66: 237-242.

7 Ishikawa S, Tanaka H, Mizushima M et al. Osteoporosis due to testicular atrophy in male leprosy patients. Acta Med Okayama, 1997; 51: 279-283.

8 Ishikawa S, Ishikawa A, Yoh K et al. Osteoporosis in male and female leprosy patients. Calcif Tissue Int, 64: $144-147$.

9 Ishikawa S, Mizushima M, Furuta M et al. Leydig cell hyperplasia and the maintenance of bone volume: bone histomorphometry and testicular histopathology in 29 male leprosy autopsy cases. Int J Lepr, 2000; 68: 258 266. 
${ }^{10}$ Fujimoto D, Suzuki M, Uchiyama A et al. Analysis of pyridinoline, a cross-linking compound of collagen fibers, in human urine. J Biochem, 1983; 94: 1133-1136.

11 Robin SP, Black D, Paterson CR et al. Evaluation of urinary hydroxypyridinium crosslink measurements as resorption markers in metabolic bone disease. Eur J Clin Invest, 1991; 21: 310.

12 Gamero P, Shin WJ, Gineyts E et al. Comparison of new biochemical markers of bone turnover in late postmenopausal osteoporotic women in response to alendronate reatment. J Clin Endocrinol Metab, 1994; 79: 1693-1700.

13 Kawana K, Kushida K, Takahashi M et al. The effect of menopause on biochemical markers and ultrasound densitometry in healthy females. Calcif Tissue Int, 1994; 55: 420-425.

14 Uebelhart D, Schlemmer A, Johansen JS et al. Effect of menopause and hormone replacement therapy on the urinary excretion of pyridinium cross-links. J Clin Endocrinol Metab, 1991; 72: 367-373.

15 Nishimoto SK, Price PA. Secretion of the vitamin K-dependent protein of bone by rat osteosarcoma cells. Evidence for an intracellular precursor. J Biol Chem, 1980; 255: 6579-6583.

16 Brown JP, Delmas PD, Malaval L et al. Serum bone Gla-protein, a specific marker for bone formation in postmenopausal osteoporosis. Lancet, 1984; 19: 1091-1093.

17 Kin K, Kushida K, Yamazaki K et al. Bone mineral density of the spine in normal Japanese subjects using dualenergy X-ray absorptiometry: effect of obesity and menopausal status. Calcif Tissue Int, 1991; 49: 101-106.

18 Kevin JM. The metabolism and radioimmunoassay of parathyroid hormone. JBMM, 1991; 9: 32(270)-38(276).

19 James IT. Rapid assay for hard tissue collagen cross-links using isocratic ion-pair reversed-phase liquid chromatography. J Chromatogr, 1990; 525: 43-57.

20 Bullamore JR, Gallagher JC, Wilkinson R, Nordin BEC. Effect of age on calcium absorption. Lancet, 1970; 12: 535-537.

21 Gallagher JC, Riggs BL, Eisman J et al. Intestinal calcium absorption and serum vitamin D metabolites in normal subjects and osteoporotic patients. Effect of age and dietary calcium. J Clin Invest, 1979; 64: 729-736.

22 Lips P, Netelenbos JC, Jongen MJ et al. Histomorphometric profile and vitamin D status in patients with femoral neck fractures. Metab Bone Dis Relat Res, 1982; 4: 85-93.

23 Komar L, Nieves J, Cosman F et al. Calcium homeostasis of an elderly population upon admission to a nursing home. J Am Geriatr Soc, 1993; 41: 1057-1064.

24 Riggs BL, Melton III LJ. Involutational osteoporosis, N Engl J Med, 1986; 314: 1676-1686.

${ }^{25}$ Francis RM, Peacock M, Aaron JE et al. Osteoporosis in hypogonadal men: role of decreased plasma 1,25dihydroxyvitamin D, calcium malabsorption, and low bone formation. Bone, 1986; 7: 261-268.

26 Jackson JA, Kleerekoper M, Parfitt AM et al. Bone histomorphometry in hypogonadal and eugonadal men with spinal osteoporosis. J Clin Endocrinol Metab, 1987; 65: 53-58.

27 Morishima A, Grumbach MM, Simpson ER et al. Aromatase deficiency in male and female siblings caused by a novel mutation and the physiological role of estrogens. J Clin Endocrinol Metab, 1995; 80: 3689-3698.

${ }^{28}$ Carani C, Qin K, Simoni M et al. Effect of testosterone and estradiol in a man with aromatase deficiency. $N$ Engl $J$ Med, 1997; 337: 91-95.

29 Bilezikian JP, Morishima A, Bell J, Grumbach MM. Increased bone mass as a result of estrogen therapy in a man with aromatase deficiency. N Engl J Med, 1998; 339: 599-603.

30 Smith EP, Boyd J, Frank GR et al. Estrogen resistance caused by a mutation in the estrogen-receptor gene in a man. N Engl J Med, 1994; 20: 1056-1061. 